\title{
A single-centre experience of scrub typhus in a tertiary care centre - a re-emerging infection
}

\author{
Sahoo D. ${ }^{1 *}$, Maikap D. ${ }^{2}$, Mohanty L. ${ }^{3}$, Patro S. ${ }^{4}$, Sekhar Panda S. ${ }^{5}$, Prasad Mohanty A. ${ }^{6}$ \\ DOI: https://doi.org/10.17511/ijmrr.2020.i01.09 \\ 1* Debananda Sahoo, Assistant Professor, Department of Internal Medicine, All India Institute of Medical Sciences, Bhubaneswar, Odisha, \\ India. \\ 2 Debasish Maikap, Senior Resident, Department of Rheumatology, Kalinga Institute of Medical Sciences, Bhubaneswar, Odisha, India. \\ 3 Lalatendu Mohanty, Professor, Department of Internal Medicine, Kalinga Institute of Medical Sciences, Bhubaneswar, Odisha, India. \\ ${ }^{4}$ Shubhransu Patro, Professor, Department of Internal Medicine, Kalinga Institute of Medical Sciences, Bhubaneswar, Odisha, India. \\ 5 Sudhansu Sekhar Panda, Professor, Department of Internal Medicine, Kalinga Institute of Medical Sciences, Bhubaneswar, Odisha, India. \\ ${ }^{6}$ Ambika Prasad Mohanty, Professor, Department of Internal Medicine, Kalinga Institute of Medical Sciences, Bhubaneswar, Odisha, India.
}

Background: Scrub typhus is a documented disease in Himachal Pradesh, but there have been few clinico-epidemiological studies in this area. The present study is done with IgM ELISA as a diagnostic test which has higher sensitivity and specificity as most of the previous studies had used Weil Felix test as a diagnostic test. Methodology: This was a prospective observational study. All the patients more than 18 years of age with positive IgM ELISA for scrub typhus with/without eschar were included. The clinical profile was observed. IgM scrub typhus was done by ELISA. Results: Total of 39 patients were observed between July 2016 to Dec 2016. Maximum patients were observed in August, September, and October. Fever with Headache was the most common presenting complaint. Eschar was present in $10 \%$ patients. Complications were seen in $76.92 \%$. The mortality rate was 0 $\%$. Conclusion: The varied presentations and high rate of complications require a high index of suspicion for Scrub Typhus. The general physicians should be sensitized for the early diagnosis and treatment to reduce morbidity and mortality.

Keywords: Scrub Typhus, Eschar, Orientia tsutsugamushi, Acute febrile illness

Corresponding Author

Debananda Sahoo, Assistant Professor, Department of Internal Medicine, All India Institute of Medical Sciences, Bhubaneswar, Odisha, India. Email: drdebanandasahoo@gmail.com

\section{How to Cite this Article}

Sahoo D, Maikap D, Mohanty L, Patro S, Panda SS, Mohanty AP. A single-centre experience of scrub typhus in a tertiary care centre - a re-emerging infection. Int J Med Res Rev. 2020;8(1):57-62.

Available From

https://ijmrr.medresearch.in/index.php/ijmrr/article/ view/1101
To Browse

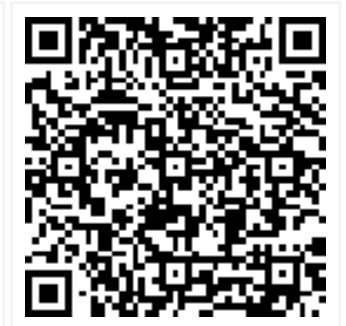

Manuscript Received 2020-01-10

Conflict of Interest No
Review Round 1 2020-01-20

Funding

$\mathrm{Nil}$
Review Round 2 2020-01-25

Ethical Approval Yes
Review Round 3

Accepted 2020-01-28

C 2020 by Debananda Sahoo, Debasish Maikap, Lalatendu Mohanty, Shubhransu Patro, Sudhansu Sekhar Panda, Ambika Prasad Mohanty and Published by Siddharth Health Research and Social Welfare Society. This is an Open Access article licensed under a Creative Commons Attribution 4.0 International License https://creativecommons.org/licenses/by/4.0/ unported [CC BY 


\section{Introduction}

Scrub typhus is highly under-diagnosed because of its varied and nonspecific clinical presentation, less access to specific diagnostic modalities in most of the areas, and less index of suspicion by the clinicians even if its high endemicity in our country. Its presentation varied from a nonspecific febrile illness with constitutional symptoms like fever, rash, myalgias and headache to a disease process manifesting with multiple organ dysfunctions involving various organs such as kidney (acute renal failure), liver (hepatitis), lungs (acute respiratory distress syndrome), central nervous system (meningitis), or with circulatory collapse with haemorrhagic features. The causative organism for Scrub typhus is Orientia tsutsugamushi (Rickettsia tsutsugamushi). In India, Scrub typhus and other Rickettsial diseases have been present since very long duration. It was more prevalent in Southeast Asia During World War II, having a major contribution towards morbidity and mortality but the incidence was declined considerably in the later decades and was believed to have disappeared from many parts of India. However, there is a resurgence of scrub typhus in several parts of India, including South India, supported by various recent reports. There are many epidemics of scrub typhus which have been reported from North, East and South India [1-9]. There are few case reports of scrub typhus from Odisha in the literature, though the cases sporadically recently with a rising tendency in incidence are commonly encountered. As the clinical diagnosis is difficult and there is lack of laboratory methods for diagnostic aid in many areas, the public health importance of this disease is underestimated. The incubation period of Scrub typhus ranges from 6 to 21 days with a characteristic presentation of high-grade fever $\left(103-105^{\circ} \mathrm{F}\right)$ associated with other symptoms like headache, Nausea/Vomiting, cough, myalgia, conjunctival suffusion and gastrointestinal symptoms. An eschar at the site of tick bite associated with regional lymphadenopathy and a maculopapular rash helps in the diagnostic process. Mortality rates is high among untreated patients ranging from $0-30 \%$ and varied depending on the patient's age and region of infection. If other severe complications such as ARDS/Meningitis arise, mortality may still be higher. Most of the time, scrub typhus associated with various complications increasing the fatality rate. Various common complications associated with scrub typhus are hepatitis, acute kidney injury, meningoencephalitis,
Myocarditis, ARDS and septic shock. In spite of the availability of effective treatment in the form of doxycycline, azithromycin, a large number of patients develops complication with high mortality mostly because of delay in the diagnosis and late initiation of specific treatment [10]. In Odisha, there were very few clinico-epidemiological studies regarding scrub typhus is present. So, this prospective observational study was planned in one tertiary care centre of eastern India to provide knowledge regarding various aspects of scrub typhus in adults. The present study was done with IgM ELISA as a diagnostic test as it has better sensitivity and specificity.

\section{Material and Methods}

Setting: This was a tertiary care hospital-based study in the department of General Medicine, KIMS, Bhubaneswar, Odisha.

Type of study and Duration: a prospective observational study over a period of 6 months ranging from July 2016 to Dec. 2016.

Sample Size calculation: Considering of prevalence of Scrub typhus in the localities from recent past studies with a confidence interval of $95 \%$ and margin of error of $5 \%$, the sample size calculated to be as 37.44 , so total number of patients taken were 39 .

Inclusion criteria: All patients with acute febrile illness above 18 years of age admitted in the IPD with the diagnosis of Scrub Typhus with or without having an eschar.

Diagnosis of scrub typhus has been made on the basis of the following.

01. Fever with Eschar and IgM ELISA positive for Scrub Typhus

02. Fever without Eschar and IgM ELISA positive for Scrub Typhus along with the response to doxycycline.

Exclusion Criteria: Patients having other associated infection. Patient with other co-morbid conditions like chronic renal failure, chronic liver disease, any neoplastic disease etc.

Data collection: The clinical profile was observed and recorded using a detailed history of symptoms, recreation, agricultural activities, travel to endemic areas, any treatment record before admission to this hospital and a detailed physical examination and the treatment outcome were noted. Supportive 
Investigations like complete blood count, urine routine and microscopy, renal function test, hepatic function test, bleeding time, clotting time, PT/INR, rapid diagnostic test for malaria, peripheral blood smear for malarial parasites, Leptospira IgM for Leptospirosis, Widal test, serological test for dengue and blood culture were done. For scrub typhus, IgM antibodies detection was done by qualitative ELISA in serum. Radiologically chest X-ray, Ultrasonography abdomen and pelvis, and CT brain or MRI brain were done wherever indicated. If lymphadenopathy was present, fine needle aspiration cytology was done. For the diagnosis of associated complication and for the ease of comparison, the same standard definitions were used as of other studies on scrub typhus [11].

\section{"Multiple-organ dysfunction syndrome (MODS): Dysfunction of more than one organ, requiring intervention to maintain homoeostasis.}

Acute kidney injury: A rise in serum creatinine of more than $1.6 \mathrm{mg} / \mathrm{dl}$ or urine output less than 400 $\mathrm{ml} / 24$ hrs failing to improve after adequate rehydration.

Acute respiratory distress syndrome (ARDS): Bilateral alveolar or interstitial infiltrates on chest radiograph and $\mathrm{PaO} 2 / \mathrm{FiO} 2$ less than or equal to 200 $\mathrm{mmHg}$.

Hepatitis: Rise in SGOT and SGPT of more than three times the upper normal limit and/or elevation of serum bilirubin $>3$ times the upper limit of normal value.

Pancreatitis: Rise in threefold or more above the normal level of serum amylase and serum lipase level with typical pain upper abdomen of pancreatitis or radiological evidence of pancreatic involvement.

Meningitis: Altered sensorium with the feature of meningeal irritation like neck rigidity, positive Kernig's sign with elevated protein and/or polymorphic leucocytosis on CSF analysis.

\section{Disseminated intravascular coagulation (DIC):} was defined as a clinical manifestation of bleeding along with thrombocytopenia and elevated coagulation profile (raised fibrin degradation products, prolonged prothrombin time/INR, activated partial thromboplastin time-aPTT): Platelet count $<80,000 / L$ or $50 \%$ decrease in platelet count from the highest value recorded over previous 3 days.
Myocarditis: Myocarditis was defined as the presence of systolic global left ventricular wall motion abnormalities on 2D echocardiography along with ECG changes and clinical findings consistent with left ventricular dysfunction in a previously normal individual.

Shock: Systolic blood pressure of $<90 \mathrm{~mm} \mathrm{Hg}$ for at least $1 \mathrm{~h}$ despite adequate fluid resuscitation was labelled as a shock."

All patients who were diagnosed as scrub typhus or having strong clinical suspicion for scrub typhus were treated with doxycycline in the dose of $100 \mathrm{mg}$ twice daily PO for 10 days unless there is any contraindication. Where doxycycline could not use, azithromycin in a dose of $500 \mathrm{mg}$ once daily for 5 days was given. As per requirement, other supportive measures like haemodialysis, mechanical ventilation, blood transfusion, ionotropic agent etc. were given where ever required.

Data analysis: Statistical analysis was done with SPSS version 19. Statistically significant level was taken at $p \leq 0.05$ with a confidence interval of $95 \%$. All results are compiled in an excel sheet. Frequency distribution was calculated as a percentage.

Ethical clearance: Ethical clearance was obtained from the Ethics Committee of the institution prior to starting the study.

\section{Results}

This study included a total number of 39 patients of proven scrub typhus, admitted in the Medicine ward in Kalinga Institute of Medical Sciences, Bhubaneswar between July 2016 to December 2016. Patients with positive IgM ELISA report for scrub typhus were only included and observed. Out of the 39 patients 17 were females $(43.58 \%$ ) and 22 were males $(56.42 \%)$. Majority of the patients (30) were associated with agricultural works (76.92\%), either directly or indirectly, among whom males are predominant $(60 \%)$. Demographic profile of the patients can be seen in Table 1 . In the current study the number of cases started rising in July 6(15.38\%), peaked in August $12(30.77 \%)$ and September $15(38.46 \%)$, and declined thereafter in October $4(10.25 \%)$ and depicted graphically in Figure $1.33(84.61 \%)$ of all our cases occurred in three months of peak rainy season and only $16 \%$ cases occurred during rest of year which is statistically significant $(p=.000)$. 
Table-1: Demographic profile of the patients.

\begin{tabular}{|l|l|l|l|}
\hline \multicolumn{1}{|c|}{ Variable } & \multicolumn{1}{c|}{$\begin{array}{c}\text { Total } \mathbf{N = 3 9} \\
(\%)\end{array}$} & \multicolumn{1}{|c|}{$\begin{array}{c}\text { Female } \mathbf{N}= \\
\mathbf{1 7}(\%)\end{array}$} & \multicolumn{1}{c|}{$\begin{array}{c}\text { Male } \mathbf{N}=\mathbf{2 2} \\
(\%)\end{array}$} \\
\hline $\begin{array}{l}\text { Sex } \\
\text { Farmers }\end{array}$ & $39(100 \%)$ & $17(43.58 \%)$ & $22(56.42 \%)$ \\
\hline Others & $30(76.92 \%)$ & $12(40 \%)$ & $18(60 \%)$ \\
\hline
\end{tabular}

Fever was the main complaint, present in all 39 (100\%) patients followed by headache $12(30.76 \%)$, shortness of breath $7(17.94 \%)$, vomiting $30(76.92 \%)$, cough $9(23.07 \%)$, altered sensorium $4(10.25 \%)$, decreased urine output $10(25.64 \%)$ among patients (Table 2). At the time of admission clinical signs noted were hypotension 6(15.38\%), icterus 32(82.05\%), eschar 3(7.69\%), skin rash $3(7.69 \%)$. In the present study the eschar was found in $7.69 \%$ of the patients which was very low in comparison to $41.7 \%, 46 \%$ from studies done by Dass et al at Meghalaya [12] and M. Vivekanandan et al study [1] at Pondicherry, while most studies of the Oriental countries had eschar in 60-70\% patients. The probable explanation for the less frequency of eschar in our area i.e. India specifically in Odisha is variation in cutaneous immunity as well as dark skin of the patients. The active search must be carried out to find the eschar at unusual locations, as this is a painless lesion.

Table 2: Signs and symptoms at presentation.

\begin{tabular}{|l|l|l|l|}
\hline \multicolumn{1}{|c|}{ Variable } & \multicolumn{1}{|c|}{$\begin{array}{c}\text { Total N=39 } \\
(\%)\end{array}$} & \multicolumn{1}{|c|}{$\begin{array}{c}\text { Female N= } \\
\mathbf{1 7}(\%)\end{array}$} & \multicolumn{1}{|c|}{$\begin{array}{c}\text { Male N=22 } \\
(\%)\end{array}$} \\
\hline Fever & $39(100 \%)$ & $17(100 \%)$ & $22(100 \%)$ \\
\hline $\begin{array}{l}\text { Cough } \\
\text { breath }\end{array}$ & $9(23.07 \%)$ & $4(23.52 \%)$ & $5(22.72 \%)$ \\
\hline Altered sensorium & $4(10.25 \%)$ & $1(5.88 \%)$ & $3(13.63 \%)$ \\
\hline $\begin{array}{l}\text { Decreased } \\
\text { urination }\end{array}$ & $10(25.64 \%)$ & $4(23.52 \%)$ & $6(27.27 \%)$ \\
\hline Headache & $12(30.76 \%)$ & $4(23.52 \%)$ & $8(36.36 \%)$ \\
\hline Vomiting & $30(76.92 \%)$ & $14(82.35 \%)$ & $16(72.72 \%)$ \\
\hline Rash & $3(7.69 \%)$ & $0(0 \%)$ & $3(13.63 \%)$ \\
\hline SBP $\leq 90 \mathrm{~mm} \mathrm{Hg}$ & $6(15.38 \%)$ & $2(11.76 \%)$ & $4(18.18 \%)$ \\
\hline Eschar & $3(7.69 \%)$ & $0(0 \%)$ & $3(13.63 \%)$ \\
\hline
\end{tabular}

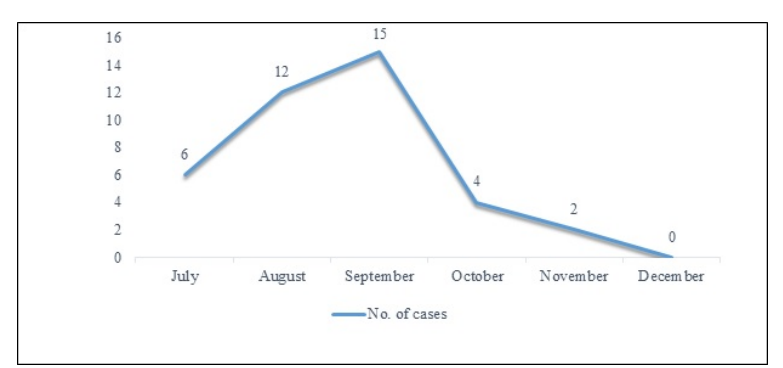

\section{Fig-1: Seasonal Disease Trend}

Complications rates were also quite high among the patients, the most common complication being hepatic dysfunction with $82.05 \%$ of patients were having, followed by ARDS (53.84\%) and MODS (48.71\%). The other common complications which were developed in our patients were renal dysfunction (25.64\%) and features of sepsis (25.64\%). $15.38 \%$ of patients came with septic shock (Table 3 ).

Table-3: Complications of Scrub Typhus.

\begin{tabular}{|l|l|l|l|}
\hline & \multicolumn{1}{|c|}{$\begin{array}{c}\text { Total } \mathbf{N}=39 \\
(\%)\end{array}$} & \multicolumn{1}{|c|}{$\begin{array}{c}\text { Female } \mathbf{N}= \\
17(\%)\end{array}$} & \multicolumn{1}{|c|}{$\begin{array}{c}\text { Male } \mathbf{N}=22 \\
(\%)\end{array}$} \\
\hline Hepatic & $32(82.05 \%)$ & $12(70.58 \%)$ & $20(90.90 \%)$ \\
\hline Renal & $10(25.64 \%)$ & $4(23.52 \%)$ & $6(27.27 \%)$ \\
\hline ARDS & $21(53.84 \%)$ & $6(35.29 \%)$ & $15(68.18 \%)$ \\
\hline MODS & $19(48.71 \%)$ & $4(23.52 \%)$ & $15(68.18 \%)$ \\
\hline Meningoencephaliti & $4(10.25 \%)$ & $1(5.88 \%)$ & $3(13.63 \%)$ \\
\hline S & & & $7(31.81 \%)$ \\
\hline Sepsis & $10(25.64 \%)$ & $3(17.64 \%)$ & $4(18.18 \%)$ \\
\hline Septic shock & $6(15.38 \%)$ & $2(11.76 \%)$ & \\
\hline
\end{tabular}

\section{Discussion}

The present study has observed that scrub typhus can have a varied clinical presentation from only fever with or without eschar to fever with MODS [13]. A total of 39 cases of Scrub typhus confirmed by IgM ELISA positive out of which $56.42 \%$ were male and $43.58 \%$ were females. The male preponderance may be associated with the local working culture of males being more involved in farming work. Maximum no. of the patients were farmers i.e. 30 (76.92\%) and only 9 (23.08\%) patients had other profession but indirectly they were also involved in farming works. Similar observations had also been observed in other Indian studies done by Sanjay $v$ et al. [14]. In the present study, $87.17 \%$ of all our cases occurred in three months of peak rainy season (August, September, and October) which was similar to the study by Sanjay Vikrant et al [14] conducted in a western Himalayan state and only $13 \%$ cases occurred during rest of year which is statistically significant $(p=.000)$. Most of the Scrub Typhus symptoms are nonspecific in nature including fever, breathlessness, myalgia, headache, vomiting etc and they usually don't vary among subjects of various different studies. In the present study, fever was the main symptom which was present in $100 \%$ of patients accompanied by headache, vomiting, cough, shortness of breath $[14,15,16]$. An eschar at the 
Site of the bite is an important clue for diagnosis but there is considerable variation in the presence of eschar among various studies. In the present study the eschar was found in $7.69 \%$ of the patients which was very low in comparison to $41.7 \%, 46 \%$ from studies done by Dass et al at Meghalaya [12] and M. Vivekanandan et al [1] at Pondicherry, while most studies of the Oriental countries had eschar in $60-70 \%$ patients. Recent studies from northern India $[17,18]$ and southern India $[19,20,21]$ have also the similarly low level of prevalence of Eschar in their populations. The probable explanation for the less frequency of eschar in our area i.e. India specifically in Odisha is variation in cutaneous immunity as well as dark skin of the patients. Also, patients were unaware and ignore the presence of eschar leading to missing of that. Very rarely a detailed physical examination was done in search of an eschar [22] which also contributes to the low prevalence of eschar. The active search must be carried out to find the eschar at unusual locations, as this is a painless lesion. The rate of various complications is high in patients suffering from Scrub Typhus and the incidence is further high if there is a delay in diagnosis and treatment. In the present study, a large no. of patients has presented with complications probably due to delay in suspicion and diagnosis. Maximum no. of patients had Hepatic dysfunction with elevated liver enzymes i.e. $32(82.05 \%)$ which is similar to the studies conducted by Mahajan et al [6] and Jim et al [16]. A study conducted by Varghese GM et al showed that the incidence of ARDS was $43.5 \%$ which was similar to the current study with the incidence of $53.84 \%$ [23]. The incidence of Renal dysfunction was $25.64 \%$ in the present study which is comparable to that of Varghese et al with $13 \%$ and the result of the study by Sanjay Vikrant et al [14] with $35 \%$ but much lower than the result reported by Mahajan et al i.e.64.4\% [18]. Out of 39 patients, about half $(48.71 \%)$ were presented with MODS in the current study however the incidence of MODS in other studies like that conducted by Varghese GM et al was about 33.8\% [23] which was quite comparable. In this study, altered sensorium was present in $10.25 \%$ patients with GCS below 15 . In a study conducted by George M. Varghese et al [23] at Christian Medical College, Vellore has reported meningitis or meningoencephalitis in $19.2 \%$ which is comparable to result of the current study i.e. $10.25 \%$. The mortality rate was considerably varied in different studies but the reasons for this have not been studied extensively. Overall, there is a decreasing tendency of case fatality rate due to
Scrub typhus as reports shown that scrub typhus outbreak in northern India had a mortality rate of $17.2 \%$, in 2004 which subsequently decreased to $14 \%$ in 2006[24]. In the present study, the case fatality rate was nil.

\section{Conclusion}

Scrub typhus is now a re-emerging infection in our country especially in Odisha with variable clinical manifestation from subclinical illness to severe illness making it difficult to differentiate from other infections. The clinical course also variable among different patients presenting with multiple organ system involvements, which can be serious enough to be fatal, unless diagnosed early and treated appropriately. Hence, to reduce the morbidity and mortality from Scrub typhus, all cases of acute febrile illness during this period i.e. rainy season should be evaluated for scrub typhus so that early treatment and other interventions can be possible.

\section{What this study adds to existing Knowledge}

This study can provide an insight to the present knowledge of the clinicians about the Scrub typhus in the form of 1 . The seasonal variation of occurrence and 2. About common complications which will help them for early suspicion and treatment of the patients suffering from Scrub typhus, thereby limiting the complication and mortality rates.

\section{Author's contribution}

\section{Dr. Debananda Sahoo, Dr. Debasish Maikap, Dr. Lalatendu Mohanty, Dr. Shubhransu Patro, Dr. Sudhansu Sekhar Panda, Dr. Ambika Prasad Mohanty have contributed equally in the writing and preparation of the manuscript.}

\section{Acknowledgements}

Authors are extremely thankful to the staffs of Department of General Medicine, KIMS, Bhubaneswar, Orissa for their support.

\section{Reference}

01. Vivekanandan M, Mani A, Priya YS, Singh AP, Jayakumar S, Purty S. Outbreak of scrub typhus in Pondicherry. J Assoc Physicians India. $2010 ; 58 ; 24-28$.

[Crossref] 
02. Jayaprakash V, Vamsikrishna M, Indhumathi $E$, Jayakumar M. Scrub typhus-associated acute kidney injury- A study from a South Indian Tertiary Care Hospital. Saudi J Kid Dis Transplantat. 2019;30(4)883-890. Available from:

[Article] [Crossref]

03. Mathai E, Lloyd G, Cherian T, Abraham OC, Cherian AM. Serological evidence for the continued presence of human rickettsioses in southern India. Ann Trop Med Parasitol. 2001;95(4)395-398.

doi: [Article] [Crossref]

04. Varghese G, Abraham O, Mathai D, Thomas K, Aaron $\mathrm{R}$, Kanitha $\mathrm{M}$, et al. Scrub typhus among hospitalised patients with febrile illness in South India- Magnitude and clinical predictors. J Infect. 2006;52(1)56-60.

doi: [Article] [Crossref]

05. Kamarasu K, Malathi M, Rajagopal V, Subramani K, Jagadeesh ramasamy D, Mathai E. Serological evidence for wide distribution of spotted fevers and typhus fever in Tamil Nadu. Indian J Med Res. 2007;126(2)128-130.

[Crossref]

06. Mahajan SK, Rolain J-M, Sankhyan N, Kaushal RK, Raoult D. Pediatric scrub typhus in Indian Himalayas. Indian J Pediatr. 2008;75(9)947-49. doi: [Article] [Crossref]

07. Somashekar HR, Moses PD, Pavithran S, Mathew LG, Agarwal I, et al. Magnitude and features of scrub typhus and spotted fever in children in India. J Trop Pediatr. 2006;52(3)228-29. doi: [Article] [Crossref]

08. Ittyachen AM. Emerging infections in Kerala- a case of scrub typhus. Natl Med J India. 2009;22(6)333-334.

[Crossref]

09. Sharma A, Mahajan S, Gupta ML, Kanga A, Sharma V. Investigation of an outbreak of scrub typhus in the himalayan region of India. JPN J Infect Dis. 2005;58(4)208-210.

[Crossref]

10. Jang MO, Jang HC, Kim UJ, Ahn JH, Kang SJ, Jung SI, et al. Outcome of intravenous azithromycin therapy in patients with complicated scrub typhus compared with that of doxycycline therapy using propensity-matched analysis. Antimicrob Agents Chemother. 2014;58(3)1488-1493.

doi: [Article] [Crossref]
11. Narvencar KP, Rodrigues $S$, Nevrekar RP, Dias L, Dias, $L$, et al. Scrub typhus in patients reporting with acute febrile illness at a tertiary health care institution in Goa. Indian J Med Res. 2012;136(6)1020-1024.

[Crossref]

12. Dass $R$, Deka NM, Duwarah SG, Barman $H$, Hoque $R$, Mili $D$, et al. Characteristics of pediatric scrub typhus during an outbreak in the North Eastern region of India- Peculiarities in clinical presentation, laboratory findings and complications. Indian J Pediatr. 2011;78(11)1365 1370. doi: [Article] [Crossref]

13. Rathi NB, Rathi AN, Goodman $M H$, et al. Rickettsial diseases in central India- proposed clinical scoring systemfor early detection of spotted fever. Indian Pediatr. 2011;48;867-872. [Crossref]

14. Vikrant S, Dheer SK, Parashar A, Gupta D, Thakur S, Sharma A, et al. Scrub typhus associated acute kidney injury-a study from a tertiary care hospital from western Himalayan state of India. Renal Fail. 2013;35(10)1338-43. doi: [Article] [Crossref]

15. Sirisanthana V, Puthanakit T, Sirisanthana T. Epidemiologic, clinical andlaboratory features of scrub typhus in thirty Thai children. Pediatr Infect Dis J. 2003;22(4)341-345.

[Crossref]

16. Jim WT, Chiu NC, Chan WT, Ho CS, Chang JH, Huang SY, et al. Clinical manifestations, laboratory findings and complications of pediatric scrub typhus in eastern Taiwan. Pediatr Neonatol. 2009;50(3)96-101. doi: [Article] [Crossref]

17. Khan SA, Dutta P, Khan AM, Topno R, Borah J, Chowdhury $\mathrm{P}$, et al. Re-emergence of scrub typhus in Northeast India. Int J Infect Dis. 2012;16(12)e889-e890. doi: [Article] [Crossref]

18. Mahajan SK, Rolain JM, Kashyap R, Bakshi D, Sharma V, Prasher BS et al. Scrub typhus in Himalayas. Emerg Infect Dis. 2006;12(10)15901592.

doi: [Article] [Crossref]

19. Ramyasree A, Kalawat U, Rani ND, Chaudhary A. Seroprevalence of scrub typhus at a tertiary care hospital in Andhra Pradesh. Indian J Med Microbiol. 2015;33(1)68 72.

doi: [Article] [Crossref] 
20. Varghese GM, Janardhanan J, Trowbridge $P$, Peter JV, Prakash JA, SathyendraS, et al. Scrub typhus in South India- clinical and laboratory manifestations, genetic variability, and outcome. Int J Infect Dis. 2013;17(11)e981-987.

[Crossref]

21. Mathai E, Rolain JM, Verghese GM, Abraham OC, Mathai D, Mathai $M$, et al. Outbreak of scrub typhus in Southern India during the cooler months. Ann N Y Acad Sci. 2003;990;359-364. doi: [Article] [Crossref]

22. Stephen S, Kandhakumari G, Pradeep J, Vinithra SM, Pakirsamy $K$, et al. Scrub typhus in South India- a re-emerging infectious disease. Jap J Infect Dis. 2013;66(6)552-554.

doi: [Article] [Crossref]
23. Varghese GM, Mathew A, Kumar S, Abraham OC, Trowbridge $\mathrm{P}$, Mathai E. Differential diagnosis of scrub typhus meningitis from bacterial meningitis using clinical and laboratory features. Neurol India. 2013;61(1)17-20. doi: [Article] [Crossref]

24. Kumar K, Saxena VK, Thomas TG, and Lal S. Outbreak investigation of scrub typhus in Himachal Pradesh (India). J Commun Dis. 2004;36(4)277-283.

[Crossref] 